\title{
High Security Nested PWLCM Chaotic Map Bit-Level Permutation Based Image Encryption
}

\author{
Qassim Nasir ${ }^{1}$, Hadi H. Abdlrudha ${ }^{2}$ \\ ${ }^{1}$ Department of Electrical and Computer Engineering, University of Sharjah, Sharjah, UAE \\ ${ }^{2}$ Computer Science Department, Faculty of Information Technology, Petra Private University, Amman, Jordan \\ Email: nasir@sharjah.ac.ae, halsaadi@uop.edu.jo
}

Received January 27, 2012; revised March 9, 2012; accepted July 25, 2012

\begin{abstract}
Chaotic systems produce pseudo-random sequences with good randomness; therefore, these systems are suitable to efficient image encryption. In this paper, a low complexity image encryption based on Nested Piece Wise Linear Chaotic Map (NPWLCM) is proposed. Bit planes of the grey or color levels are shuffled to increase the encryption complexity. A security analysis of the proposed system is performed and presented. The proposed method combine pixel shuffling, bit shuffling, and diffusion, which is highly disorder the original image. The initial values and the chaos control parameters of NPWLCM maps are derived from external secret key. The cipher image generated by this method is the same size as the original image and is suitable for practical use in the secure transmission of confidential information over the Internet. The experimental results of the proposed method show advantages of low complexity, and high-level security.
\end{abstract}

Keywords: Encryption; Chaos; Piecewise, Chaotic Maps; Security

\section{Introduction}

In recent years, and due to the development of multimedia and information technology, digital images are shared over the public communication networks. Therefore, there is potential risk of vulnerable access to sensitive documents. Image encryption and robust cryptographic become an essential to protect these multimedia files from leakage. Conventional cipher algorithms such as DES, IDEA. etc. are not suitable for multimedia files due to public data capacity, strong pixel correlation and high redundancy which reduces the encryption performance [1]. The chaos based multimedia files encryption is not new idea. Matthew [2] was the fist step in this line of research. Large amount of work using digital chaotic techniques to construct cryptosystems has been studied and has attracted more and more attention in the last years [3-19]. Researchers are especially interested in enhancing the chaotic generators and the diffusion stage of the cryptosystems. According to the classification of chaotic systems, the security application of chaos can be divided into analog chaotic secure communications utilizing continuous dynamical systems [3] and digital chaotic cryptosystems utilizing discrete dynamical systems.

Discrete chaotic system is easy to implement and has good statistical properties, which can be used as random number generator. Chaotic system is extremely sensitive to parameters and initial conditions. If the system parameters or initial value is seen as the key, chaotic sys- tems have become a good password system [4]. Recently some researchers such as [5,6], they used two chaotic maps to encrypt the image to enhance the security. Similarly, Ashtiyani et al. [7] also employed chaotic maps and other method to encrypt the images. Ahmad [8] introduced a new method using two logistic chaotic maps and a large enough external secret keys for image encryption. This method exhibits a high security, but they did not proof this method is robust or not to common signal processing attacks. The scheme proposed in this paper is based on two chaotic maps which can overcome the periodicity of Arnold map and is more security; besides, it is robust to the common signal attacks. The researchers in $[9,10]$, proved that logistic map, that was widely used in the encryption domain, is not enough random and uniform. Then, they propose to use other chaotic maps like Piece Wise Linear Chaotic Map (PWLCM). In [11] and [12] presents a chaos-based cryptosystem for secure transmitted images abased on a 2D chaotic map which is used to shuffle the image pixel positions, accompanied with substitution (confusion) and permutation (diffusion) operations on every block. A multiple rounds, are combined using two perturbed chaotic PWLCM maps. Indeed, to obtain better dynamical statistical properties and to avoid the dynamical degradation caused by the digital chaotic system working in a finite state, a perturbation technique is used.

The objective of this research work is specially ori- 
ented towards using Nested PWLCM map based image encryption schemes. Two enhancement measures in the system efficiency have been proposed to the main components of typical chaos-based image cryptosystems: chaotic confusion and pixel diffusion processes. In the first block confusion and shuffle of bit positions is performed, while the other block is diffused by add and shift algorithm. The resultant image is of high encryption security as diffusion is performed twice on the bit and byte levels. Bit shuffling has been introduced by other researchers such in Pixel Chaotic Shuffle (PCS) [13], Pixel Shuffle (PS) [14] but the proposed method if a low complexity one as it uses a simple NPWLCM.

The paper is organized as follows: Section 2 briefly introduces the Nested PWLCM. Section 3 describes the proposed encryption algorithm; the proposed system performance measures and security analysis are given in Section 4. And finally, we summarize our conclusions in Section 5 .

\section{Nested PWLCM Chaotic Map}

The general description of chaos is an unpredictable and random-like long-term evolution that results from deterministic nonlinear systems. The simplest class of chaotic dynamic systems is one-dimensional chaotic map of the form

$$
x_{n+1}=f\left(x_{n}, \lambda\right), n=0,1,2, \cdots
$$

where the state variable $x$ and the system parameter $\lambda$ are scalars, and $f$ is a mapping function defined in the real domain.

\subsection{The Piecewise Linear Map [15]}

This map is given by:

$$
x(n+1)=\left\{\begin{array}{ll}
B x(n)+A & x(n)<0 \\
B x(n)-A & x(n) \geq 0
\end{array} n=0,1,2, \cdots\right.
$$

where parameters $A$ and $B$ are chosen to be 1 and 1.998 respectively to generate chaos. This map is extensively used for chaos generation due to its perfect properties such as uniform invariant density function; exactness, mixing and ergodicity; exponentially decaying correlation function and simple realization in both hardware and software [16]. Since the parameter $A$ represents just a scaling factor, the stochastic properties of the generated bit sequence are dependent only on the parameter $B$, which must assume values greater than 1 for the system to be chaotic and not greater than 2 to avoid the state $x(n)$ being attracted to either $+\infty$ or $-\infty$ [17].

\subsection{The Nested Piecewise Linear Chaotic Map (NPWLCM)}

The proposed Nested Piecewise Linear Chaotic Maps are expressed as $[18,19]$ :

$$
\begin{aligned}
& x_{1}(n)= \begin{cases}x_{4}(n-1)-A & x_{4}(n-1) \geq 0 \\
x_{4}(n-1)+A & x_{4}(n-1)<0\end{cases} \\
& x_{2}(n)= \begin{cases}B x_{1}(n)-A & x_{1}(n) \geq 0 \\
B x_{1}(n)+A & x_{1}(n)<0\end{cases} \\
& x_{3}(n)= \begin{cases}B x_{2}(n)-A & x_{2}(n) \geq 0 \\
B x_{2}(n)+A & x_{2}(n)<0\end{cases} \\
& x_{4}(n)= \begin{cases}B x_{3}(n)-A & x_{3}(n) \geq 0 \\
B x_{3}(n)+A & x_{3}(n)<0\end{cases}
\end{aligned}
$$

where parameters $A$ and $B$ are chaos generation parameters. The bifurcation diagram [19] of the NPWLCM shows that when the control parameter $B$ is 1.998 , chaos is still generated.

\section{Encryption System Description}

For image encryption, 2D or higher-dimensional chaotic maps are naturally employed for a reason that the image can be considered as a 2D array of pixels. Let's assume that the size of the plain image is $N \times \mathrm{M}$. If the image is of gray levels then each pixel can be represented by $2^{G}$, where $G(=8)$ is the number of bits per pixel. If the image is of a color format, then it can represented by 3D dimension array of Red, Green, and Blue (RGB) levels. Since images are composed of finite lattice called pixels, the domain of the map $f($.) is changed to the discretized form Image permutation can be achieved through shuffling the position of image bits and then pixels. It is necessary to introduce a diffusion mechanism after the bit permutation stage. The idea is to spread the influence of every single pixel over the entire image. The complexity evaluation is important to image encryption as well since it always indicates the feasibility of encryption schemes. Some special attentions should be given in terms of computational speed, size and quality of the encrypted images. The block diagram is composed of two processes: chaotic confusion and pixel diffusion as shown in Figure 1.

In confusion module, each bit position at each column in bit-plane is shuffled by using the pseudorandom sequence which is generated by NPWLCM chaotic map. The change in the bit position at each pixel will modify the pixel value. Thus the security of confusion module is significantly improved due to introducing of diffusion effect throughout the bit-level permutation operation. Moreover, the overall security - level is further enhanced by introducing another diffusion operation at the pixellevel by using a simple Add and shift operations. To achieve a satisfactory level of security the confusiondiffusion operations are repeated $(8 \times m \times n$ where $m$ and 
$n$ are the image width and high respectively) rounds iteration.

The proposed image encryption is summarized by the following steps:

1) The proposed image encryption process utilizes a 96 bit external secret key. This key is partitioned into three 32 blocks. The chaotic sequence generation control parameters $(A, B)$, and the initial condition $x(0)$ are derived from this secret key blocks.

2) Generate four chaotic bit sequences by NPWLCM using formulas $(3-6)$. XOR post processing is used to generate these random bit sequences.

3) Sort four chaotic binary sequences and generate four new integer sequences by ordinal numbers corresponding to the original sequences, such as

$$
\left[s q_{k, i}(i, j)\right]_{k=0, i=1}^{N-1,4}=\operatorname{sort}\left(\left[x_{k, i}\right]_{k=0, i=1}^{N-1,4}\right) .
$$

4) Each bit-plane is shuffled separately by using the sorting sequences generated in previous step as shown in Figure 2. Thus the pixel value (Grey Levels bits, R-level bits, G-level bits, Blue level bits) matrices are column shuffled by using the indexing generated chaotic sequences. So each level will be shuffled using the following formula by assuming the level matrix defined as

$$
\begin{aligned}
& {[\mathbf{B}(i, j)]_{i=0, j=0}^{M-1,8 N-1}:} \\
& \left(\left[\mathbf{B}^{\prime}(i, j, k)\right]_{i=0, j=0, k=1}^{M-1, N-1,8}=\left\{\begin{array}{l}
B(:, s q 1, k) k=1,2 \\
B(:, s q 2, k) k=3,4 \\
B(:, s q 3, k) k=5,6 \\
B(:, s q 4, k) k=7,8
\end{array}\right\}\right.
\end{aligned}
$$

The shuffled bit-planes are shown in Figure 2.

5) The permuted image is achieved by combining all the 8 shuffled and confused bit-planes together

6) The pixels (Grey, or RGB) value will be diffused using the following Add-Shift formula

$$
\begin{gathered}
\text { mask }=T_{i-1} \& 7 \\
X P_{i}=\left(X P_{i}+T_{i-1}\right) \bmod (256) \\
T_{i}=\left(X P_{i} \gg \text { mask }\right) \mid\left(X P_{i} \ll(8-\text { mask })\right)
\end{gathered}
$$

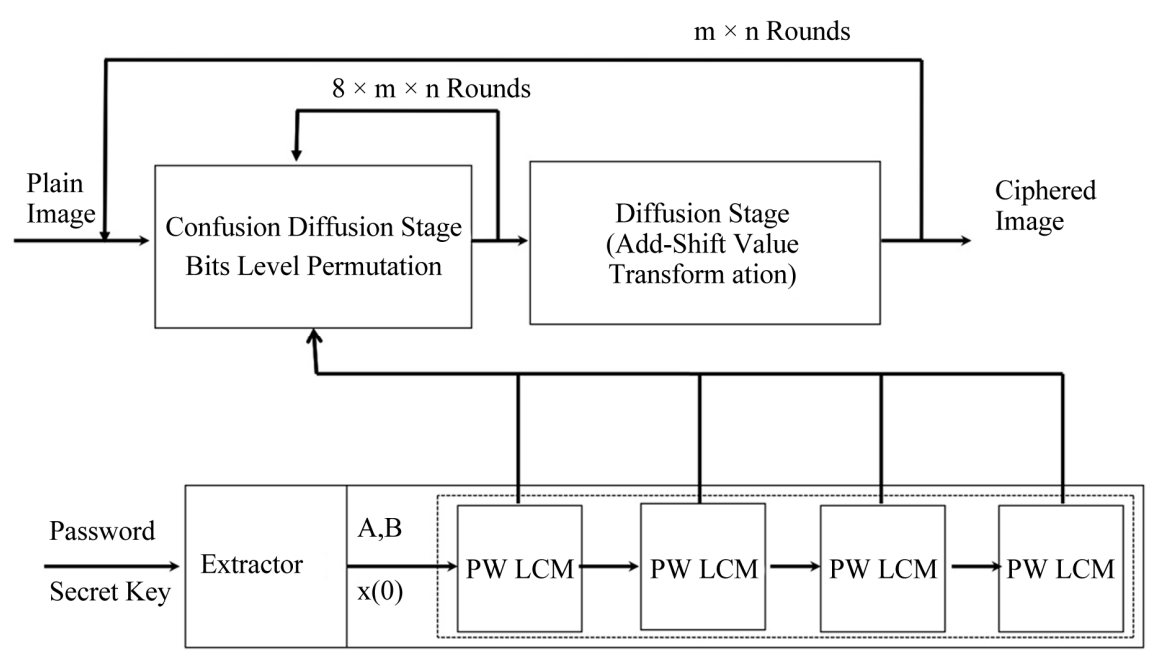

Figure 1. Standard CHAOS based image encryption.
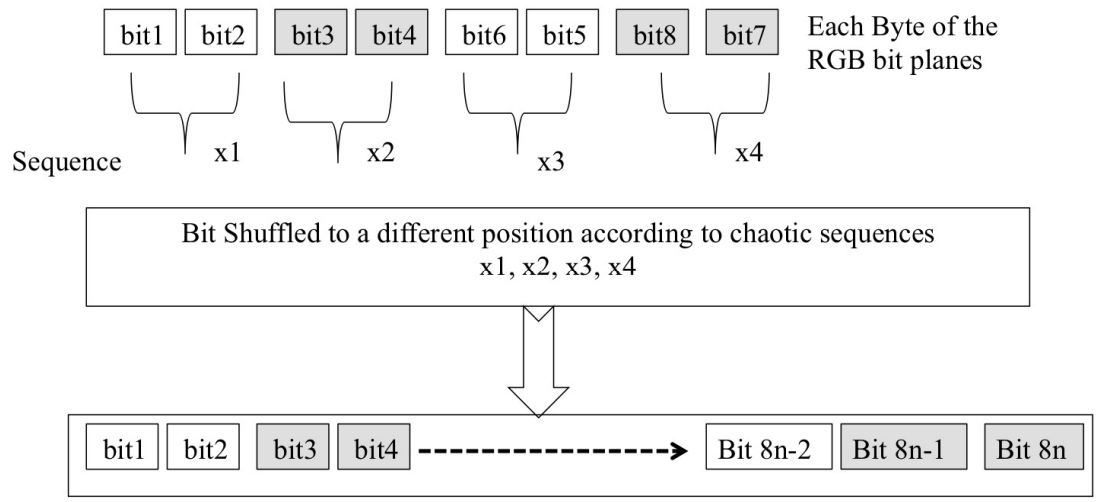

Bit Position in each Row of the RGB Bit plans

Figure 2. Bit indexing and shuffling within a row. 
where $X P_{i}$ be the value of the $(i)$ th value in the image after confusion and permutation operations, $T_{i-1}$ and $T_{i}$ be temporary values, "mask" be the number of bits to be used in right and left shifting.

\section{System Performance}

\subsection{System Performance for Grey Image Pictures}

Three test images are chosen (Lena, Baboon and camera Men) and encrypted by the proposed method, and visual test is performed as shown in Figures 3(a), (c), (e) and Figures 3(b), (d), (f), where each image is in 8 bit gray color with $256 \times 256$ pixels. By comparing the original and the encrypted images in Figure 3, there is no visual information observed in the ciphered image. In order to further demonstrate the effectiveness of proposed encryption scheme, some more tests suggested by other researchers have been carried out and the results are to be explained below [12]. An image-histogram illustrates how pixels in an image are distributed. The histograms for the original and ciphered test images are shown in Figure 4. As we can see, the histogram of the ciphered image is fairly uniform and is significantly different from that of the original images. Hence the ciphered image does not provide any clue to employ any statistical attack on the proposed image encryption procedure, which makes statistical attacks difficult.

Correlation coefficient measures the dependence of

(a)

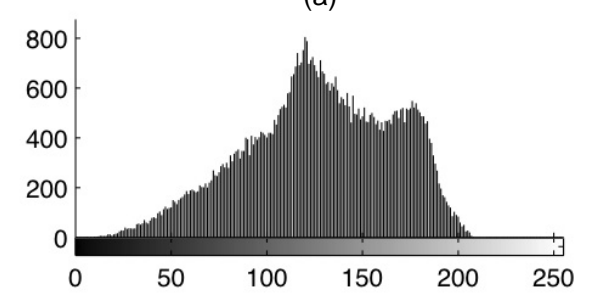

(c)

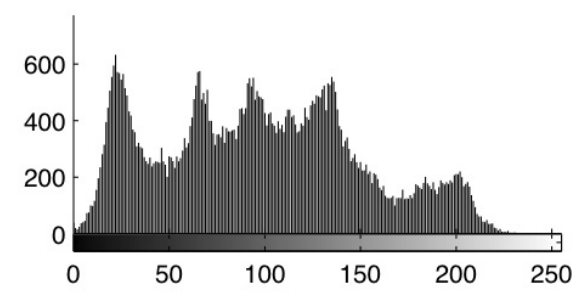

(e)

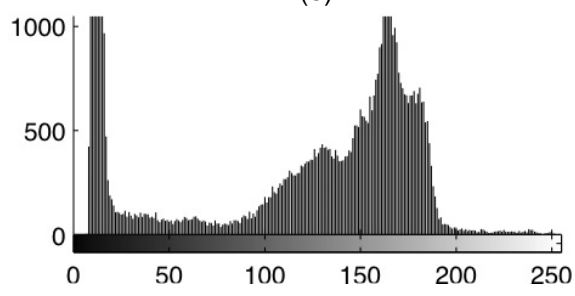

two adjacent variables at a certain direction. The more closely related these two variables are, the closer the correlation coefficient approaches 1 . Conversely, if they are less closely related, the value of correlation coefficient approaches 0 . The two variables are not related and unpredictable when the coefficient is close to 0 . For an ordinary image, each pixel is highly correlated with its adjacent pixels either in horizontal, vertical or diagonal (a)

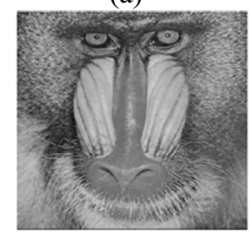

(c)

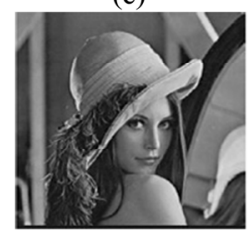

(e)

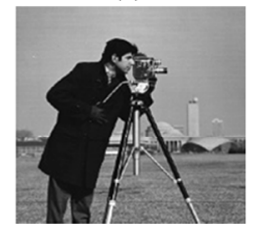

(b)

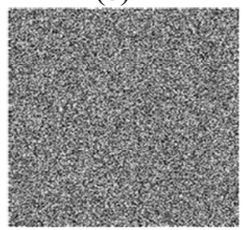

(d)

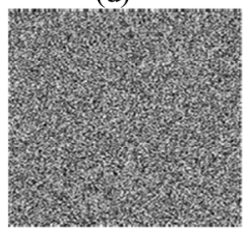

(f)

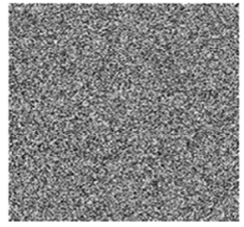

Figure 3. Images before and after Encryption.

(b)

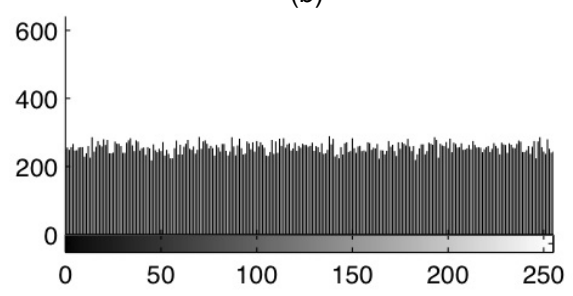

(d)

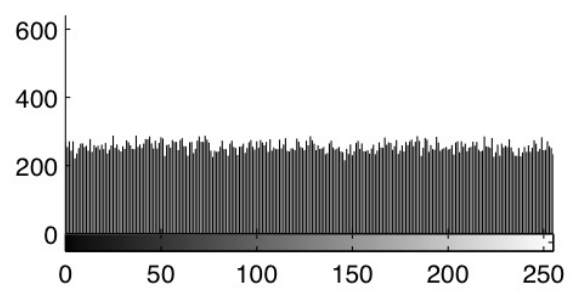

(f)

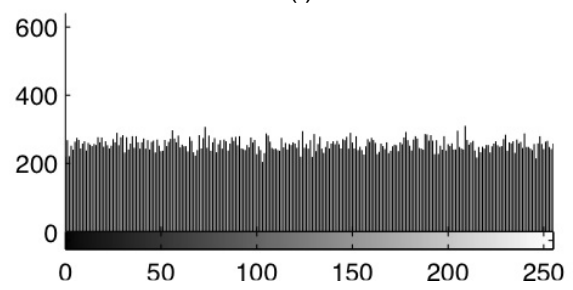

Figure 4. Histogram analysis of test images before and after encryption. 
direction. However, an efficient image cryptosystem should show sufficiently low correlation in the adjacent pixels and in all directions. The correlation distribution

(a)

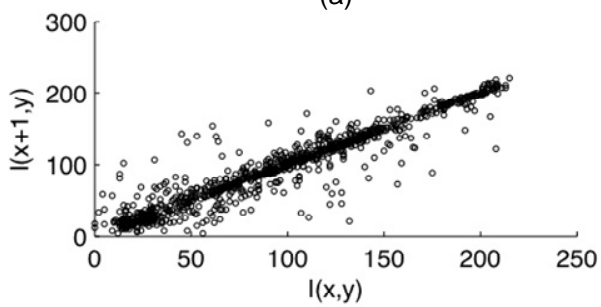

(c)

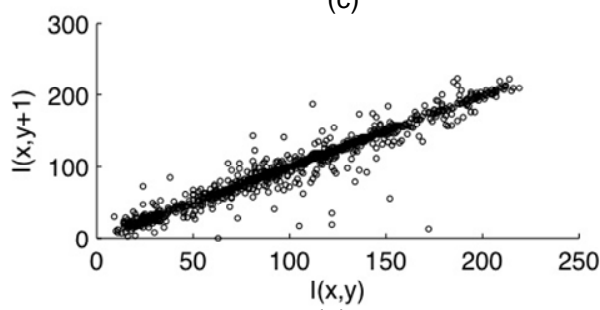

(e)

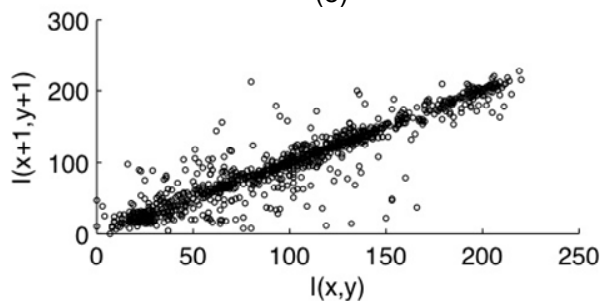

of two adjacent pixels of the plain images and the cipher images on the horizontally, vertical, and diagonal directions are shown in Figures 5-7. All adjacent pixels are

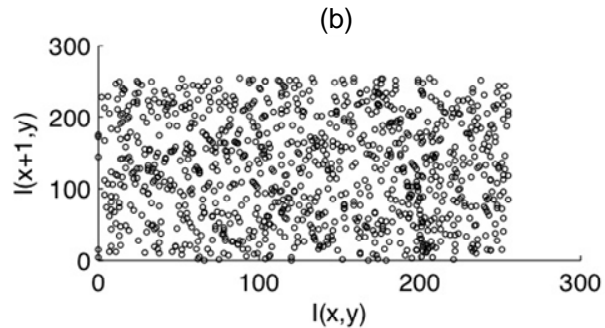

(d)

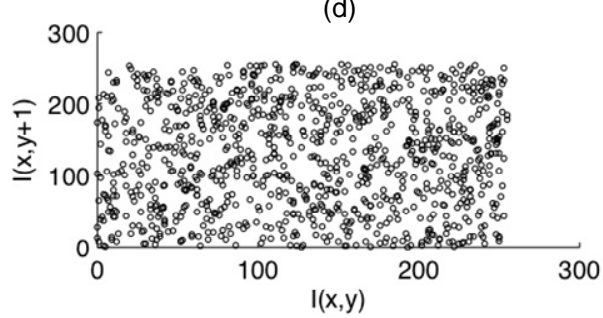

(f)

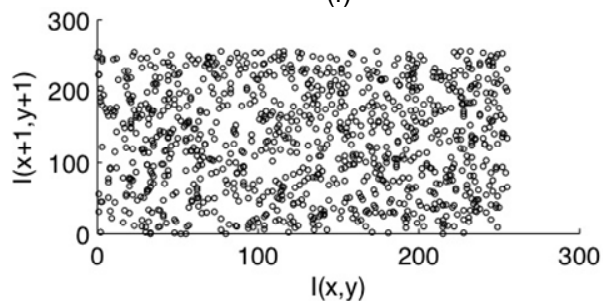

Figure 5. Correlation of two horizontal, vertical and diagonal adjacent pixels—Baboon Test Image.

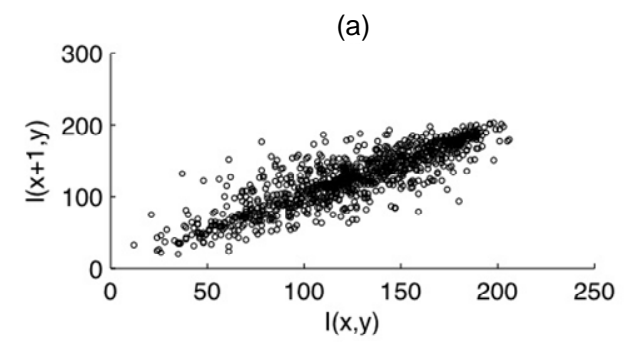

(c)

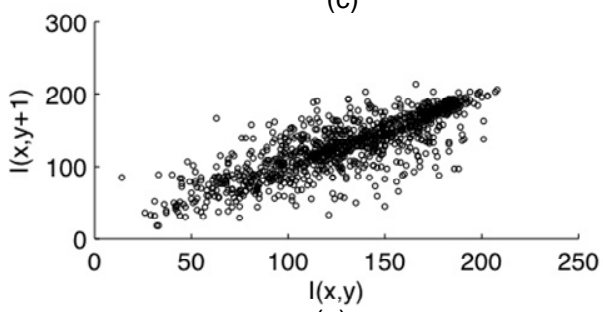

(e)

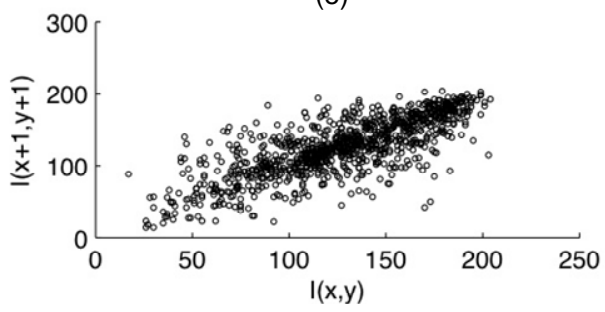

(b)

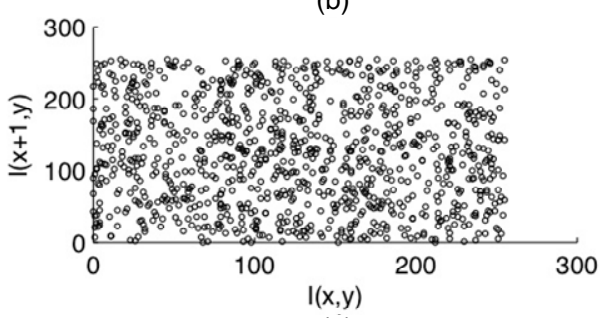

(d)

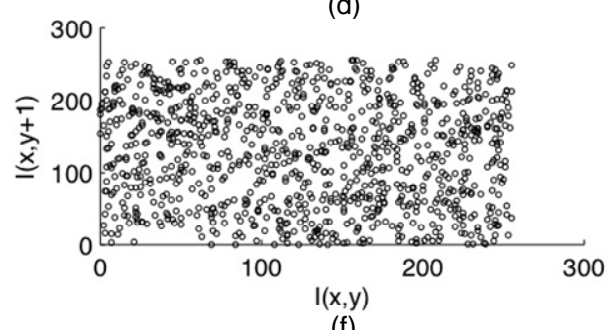

(f)

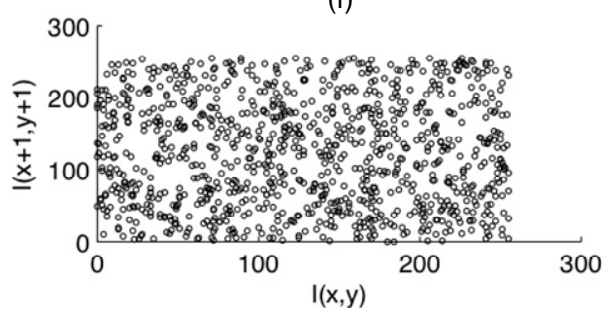

Figure 6. Correlation of two horizontal, vertical and diagonal adjacent pixels—Lena Baboon Image. 
(a)

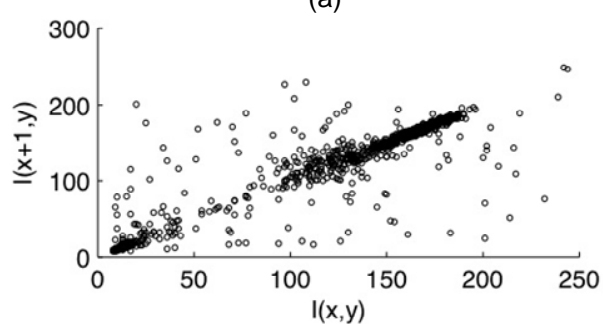

(c)

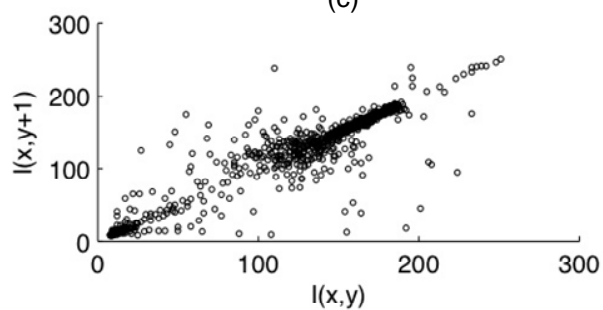

(e)

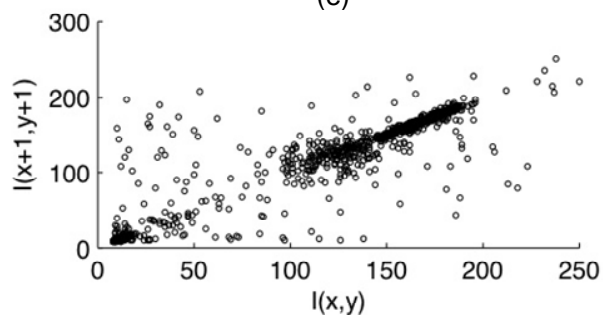

(b)

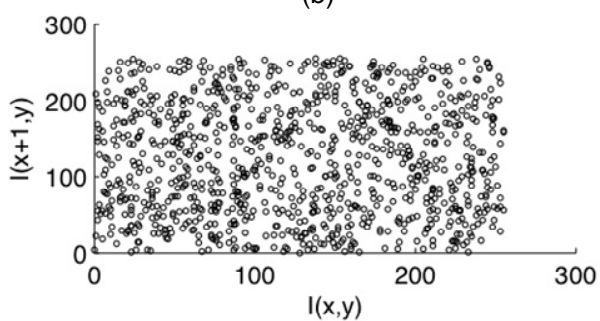

(d)

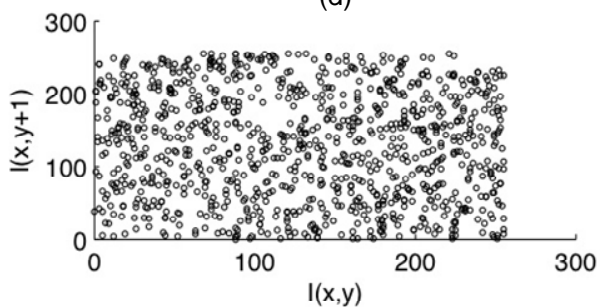

(f)

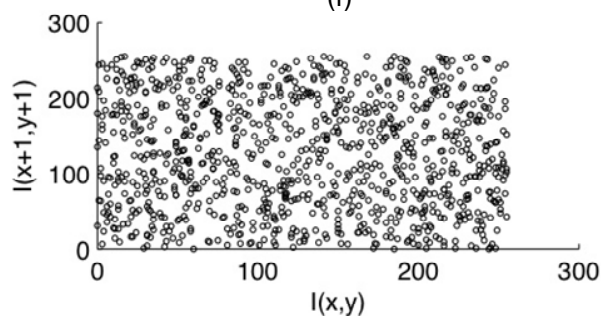

Figure 7. Correlation of two horizontal, vertical and diagonal adjacent pixels-Camera Test Image.

selected and then the pixel value on the location $(x+1, y)$ over the value on $(x, y)$ in the case of horizontal direction. Similar tests are done for pixel vale on $(x, y+1)$ over $(x$, $y$ ) for vertical, and pixel value on $(x+1, y+1)$ over $(x, y)$ in case of diagonal as shown in the Figures 5-7 for the sampled images (Lena, baboon, and camera man). To quantify and compare the correlations of adjacent pixels, the correlation coefficient $r_{x y}$ of each adjacent pair is calculated for the plain and ciphered images using the following formulas:

$$
\begin{gathered}
r_{x y}=\frac{\operatorname{cov}(x, y)}{\sqrt{D_{x}} \sqrt{D_{y}}} \\
\operatorname{cov}(x, y)=E(x-E(x))(y-E(y)) \\
E(x)=\frac{1}{L} \sum_{i=1}^{L} x_{i} \\
D_{x}=\frac{1}{L} \sum_{i=1}^{L}\left(x_{i}-E(x)\right)^{2}
\end{gathered}
$$

where $x$ and $y$ are gray scale values of two adjacent pixels in the image, and $L$ denotes the total number of samples (number of pixels in the image).

The results of the correlation coefficients for horizontal, vertical and diagonal adjacent pixels for the plain image and its cipher image are given in Table 1. It is clear from Figures 5-7 and Table 1 that the strong cor-
Table 1. Correlation coefficients for two adjacent pixels in the original and encrypted images.

\begin{tabular}{ccccccc}
\hline Corr. & \multicolumn{2}{c}{ Baboon } & \multicolumn{2}{c}{ Lena } & \multicolumn{2}{c}{ Camera } \\
\hline & Orig.1 & Encry. & Orig.1 & Encry. & Orig.1 & Encry. \\
Horiz. & 0.846 & -0.020 & 0.9471 & -0.0159 & 0.9201 & 0.0202 \\
Vert. & 0.811 & -0.044 & 0.9665 & -0.020 & 0.9443 & -0.077 \\
Diag.1 & 0.717 & -0.033 & 0.899 & 0.014 & 0.907 & -0.050 \\
\hline
\end{tabular}

relation between adjacent pixels in plain image is greatly reduced in the cipher image produced by the proposed chaos based encryption scheme.

Two common measures can be used to measure encryption performance such as number of pixels change rate (NPCR) and the unified average changing intensity (UACI) which measures the normalized mean difference between the plane and ciphered images. Let the plane, and the ciphered image denoted by $P$ and $C$ [12]. Define a bipolar array, $D$, with the same size as the images $P$ and $C . D(i, j)$ is determined as follows:

$$
D(i, j)= \begin{cases}1 & \text { if } P(i, j) \neq C(i, j) \\ 0 & \text { elsewhere }\end{cases}
$$

The NPCR is defined as

$$
\mathrm{NPCR}=\frac{\sum_{i, j} D(i, j)}{M \times N} \times 100
$$


where $M$ and $N$ are the width and height of the $P$ or $C$. While The UACI measures the average intensity of differences between the plain image and the ciphered image. UACI is defined as follows [12]:

$$
\mathrm{UACI}=\frac{1}{M \times N} \sum_{i=0}^{M-1} \sum_{j=0}^{N-1} \frac{\left|C_{1}(i, j)-C_{2}(i, j)\right|}{255} \times 100
$$

Table 2 summarizes the mean value of NPCR and UACI between the original image and the ciphered image for the Grey Images. The NPCR and UACI are high enough to say that the two images are very different.

Information Entropy will be used to measure the encryption performance as it is defined to express the degree of uncertainties in the system. It is well known that the entropy $H_{m}$ of a message source $\mathrm{m}$ can be calculated as:

$$
H_{m}=\sum_{i=0}^{2^{G}-1} p\left(m_{i}\right) \log _{2}\left(\frac{1}{p\left(m_{i}\right)}\right)
$$

where $p\left(m_{i}\right)$ represents the probability of symbols $\left(m_{i}\right), G$ $=$ number of gray level $(=8)$. If a source emits $2^{8}$ symbols with equal probabilities, then source entropy $H_{m}$ is equal to 8 . Actually, given that a practical information source seldom generates random messages, in general its entropy is smaller than the ideal one. If the entropy of encrypted images is less than, but approaching eight, it will reduce the probability of successful restoration of images by interceptors. On the contrary, if the entropy is more than eight, then interceptors easily decode the encrypted
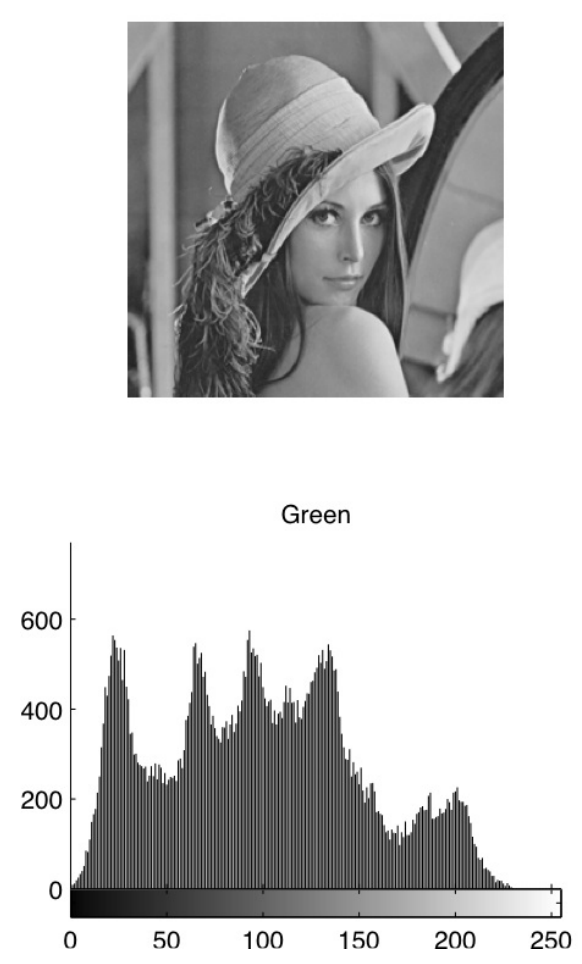

images. Table 3 shows that the entropy of the encrypted images using the proposed encryption scheme is close to theoretical value 8 . This means that information leakage in the encryption process is negligible and the encryption system is secure upon the entropy attack.

\subsection{System Performance for Color Image Pictures}

Figures 8 and 9 demonstrate that the proposed encryption algorithm change the RGB histogram to be uniform which is required by any encryption method. Table 4 summarizes the mean value of NPCR and UACI for Lena color image compared with PCS and PS. The proposed method has NPCR higher than 99.6.

Table 2. NPCR and UACI between original and ciphered images.

\begin{tabular}{lcc}
\hline Image & NPCR & UACI \\
\hline Baboon & 99.6003 & 27.3953 \\
Lena & 99.6292 & 28.505 \\
Camera Man & 99.5682 & 31.0874 \\
\hline
\end{tabular}

Table 3. Entropy of the ciphered images.

\begin{tabular}{lc}
\hline Image & Entropy \\
\hline Baboon & 7.9975 \\
Lena & 7.9975 \\
Camera Man & 7.9966 \\
\hline
\end{tabular}
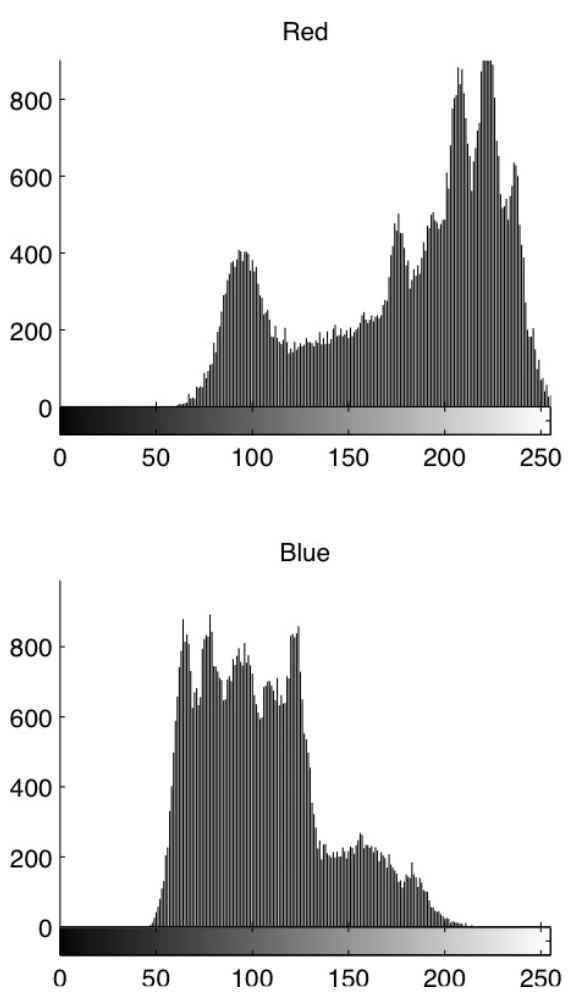

Figure 8. Lena color image and RGB intensity Histogram Analysis. 

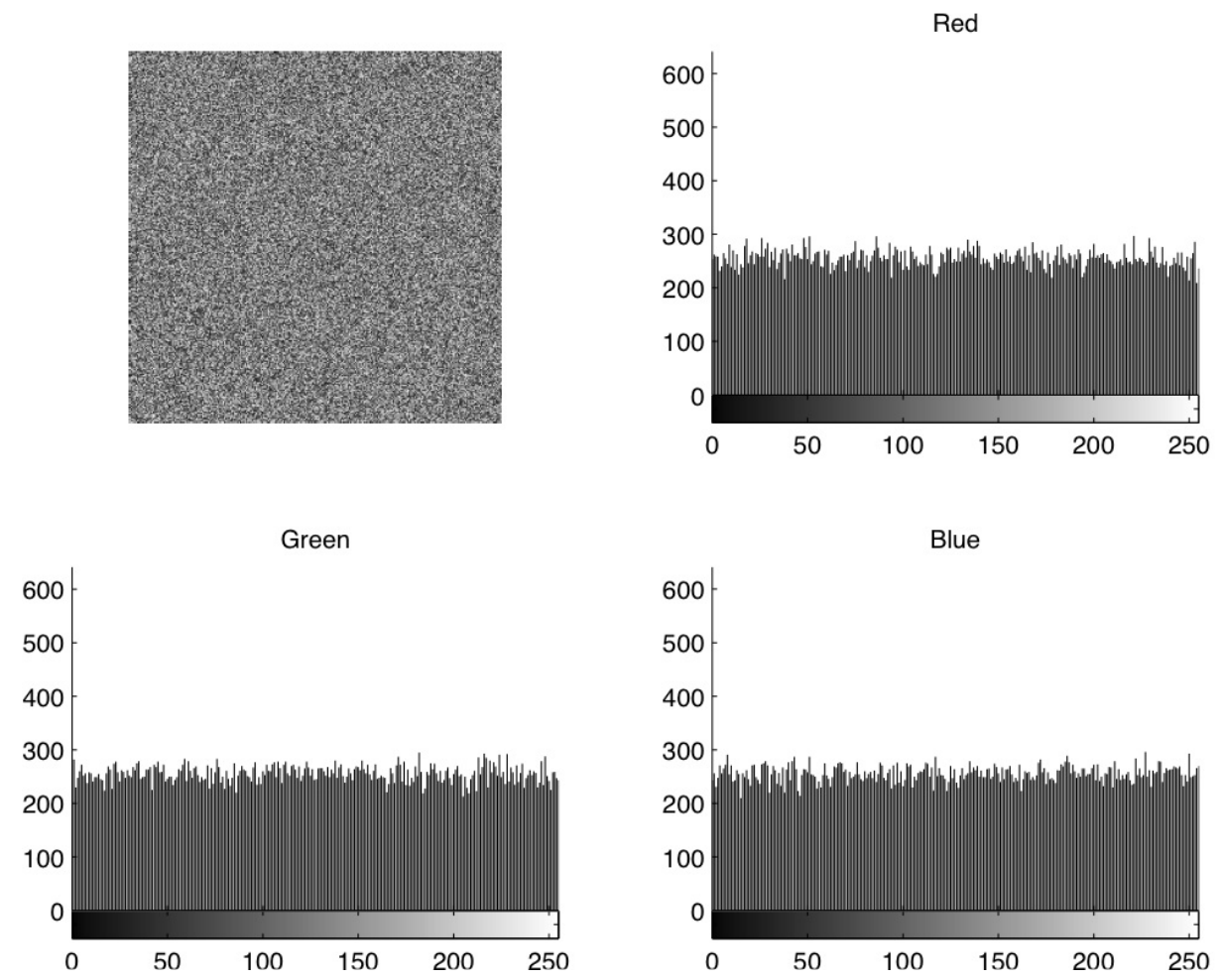

Figure 9. Encrypted Lena color image and RGB intensity histogram analysis.

Table 4. Mean value of NPCR and UACI test for color Lena Image with different encryption methods.

\begin{tabular}{lcccccc}
\hline Encryption & \multicolumn{3}{c}{ NPCR\% } & \multicolumn{3}{c}{ UACI\% } \\
\hline NPWLCM & 99.632 & 99.631 & 99.594 & 33.104 & 30.637 & 27.621 \\
PCS [13] & 99.42 & 99.6 & 99.54 & 27.78 & 27.66 & 24.94 \\
PS [14] & 99.26 & 99.45 & 99.13 & 21.41 & 23.42 & 15.08 \\
\hline
\end{tabular}

\section{Conclusion}

In this paper, an image encryption scheme based on Nested Piece Wise Linear Chaotic Map (PWLCM) is proposed. The system is stream cipher architecture. The NPWLCM chaos control parameters $(A, B)$ and initial values $x(0)$ of the maps are derived from an external password (secret key). The diffusion and confusion are conducted on the bit as well as on the pixel level and the method is applicable for both gray and color images. Detailed security analysis of the proposed scheme is presented which show a high security performance measures. The correlation coefficients of the encrypted images are below 0.07 for all test images and in all directions. In addition, high values (more than 99\%) of NPCR and UACI of $27 \%-33 \%$ prove its ability to resist against pixel changes attacks. Based on the results, we conclude that the proposed simple NPWLCM is suitable for real time secure image transmission over public networks. Implementation of the proposed encryption scheme on DSP chip is one of our future directions.

\section{REFERENCES}

[1] S. Lian, "A Block Cipher Based on Chaotic Neural Networks," Neurocomputing, Vol. 72, No. 4-6, 2009, pp. 12961301. doi:10.1016/j.neucom.2008.11.005

[2] R. Matthews, "On the Derivation of a Chaotic Encryption Algorithm," Cryptologia, Vol. 13, No. 1, 1989, pp. 29-42. doi:10.1080/0161-118991863745

[3] Z. Li and D. Xu, "A Secure Communication Scheme Using Projective Chaos Synchronization," Chaos, Solitons and Fractals, Vol. 22, No. 2, 2004, pp. 477-481. doi:10.1016/j.chaos.2004.02.019

[4] F.-Y. Wang and G.-W. Cui, "A New Image Encryption Algorithm Based on the Logistic Chaotic System," The 3rd IEEE International Conference on Computer Science and Information Technology (ICCSIT), Chengdu, 9-11 July 2010, pp. 192-194.

[5] C. M. Li and L. X. Hong, "A New Image Encryption Scheme Based on Hyperchaotic Sequences," IEEE International Workshop on Anti-Counterfeiting, Security, Identification, Xiamen, 16-18 April 2007, pp. 237-240.

[6] Y. Cheng, S. Yang and S.-F. Li, "Image Encryption of Multiple Keys Method Based on Chaotic Maps," First International Conference on Pervasive Computing Signal Processing and Applications (PCSPA), Harbin, 17-19 September 2010, pp. 891-894. doi:10.1109/PCSPA.2010.220

[7] M. Ashtiyani, P. M. Birgani and H. M. Hosseini, "ChaosBased Medical Image Encryption Using Symmetric Cryptography," The 3rd International Conference on Information and Communication Technologies: From Theory to Applications, Damascus, 7-11 April 2008, pp. 1-5. 
[8] M. Ahmad, C. Gupta and A. Varshney, "Digital Image Encryption Based on Chaotic Map for Secure Transmission," International Multimedia Signal Processing and Communication Technologies, Aligarh, 14-16 March 2009, pp. 292-295. doi:10.1109/MSPCT.2009.5164233

[9] D. Socek, S. Li, S. S. Magliveras and B. Furht, "Enhanced 1-D Chaotic Key Based Algorithm for Image Encryption," First International Conference on Security and Privacy for Emerging Areas in Communications Networks, Athens, 5-9 September 2005, pp. 406-407.

[10] G. Alvarez, F. Montoya, M. Romera and G Pastor, "Cryptanalysis of an Ergodic Chaotic Cipher," Physics Letters $A$, Vol. 311, No. 2-3, 2003, pp. 172-179. doi:10.1016/S0375-9601(03)00469-9

[11] A. Awad, S. E. Assad, Q. Wang, C. Vladeanu and B. Bakhache, "Comparative Study of 1-D Chaotic Generators for Digital Data Encryption," International Journal of Computer Science, Vol. 35, No. 4, 2008, pp. 483-488.

[12] A. Awad, "A New Chaos-Based Cryptosystem for Secure Transmitted Images," IEEE Transactions on Computers, No. 99, 2011, p. 1.

[13] C. X. Zhu, Z. G. Chen and W. W. Ouyang, "A New Image Encryption Algorithm Based on General Chen's Chaotic System," Journal of Central South University of Technology, Vol. 37, No. 6, 2006, pp. 1142-1148.
[14] C. K. Huang and H. H. Nein, "Multi Chaotic Systems Based Pixel Shuffle for Image Encryption," Optics Communication, Vol. 282, No. 11, 2009, pp. 2123-2127..

[15] A. Abid, Q. Nasir and A. Elwakil, "Implementation of an Encrypted Wireless Communication System Using Nested Chaotic Maps," International Journal of Bifurcation and Chaos, Vol. 20, No. 12, 2010, pp. 4087-4096. doi: $10.1142 / \mathrm{S} 0218127410027957$

[16] S. Li, G. Chen and X. Mou, "On the Dynamical Degradation of Digital Piecewise Linear Chaotic Maps," International Journal of Bifurcation and Chaos, Vol. 15, No. 10, 2005, pp. 3119-3151. doi:10.1142/S0218127405014052

[17] T. Addabbo, M. Alioto, A. Fort, S. Rocchi and V. Vignoli, "A Feedback Strategy to Improve the Entropy of a ChaosBased Random Bit Generator," IEEE Transactions on Circuits and Systems, Vol. 53, No. 2, 2006, pp. 326-337. doi:10.1109/TCSI.2005.856670

[18] M. Drutarovsky and P. Galajda, " Chaos-Based True Random Number Generator Embedded in a Mixed-Signal Reconfigurable Hardware," Journal of Electrical Engineering, Vol. 57, No. 4, 2006, pp. 218-225.

[19] A. M. Abid, "An Implementation of Chaotic Encryption in Wireless Voice Transmission," M.Sc. Thesis, University of Sharjah, Sharjah, 2009. 